\title{
Composición, caracterización y potencial aterogénico de aceites, grasas y otros derivados producidos o comercializados en Costa Rica
}

\author{
Monserrat Castro-Bolaños ', Carlos H. Herrera-Ramírez ${ }^{2}$, Giselle Lutz-Cruz ${ }^{2}$
}

\section{Resumen}

Las enfermedades cardiovasculares, tan comunes entre los costarricenses, están relacionadas con la ingesta de altas cantidades de lípidos capaces de ejercer una acción perjudicial en las arterias del cuerpo humano. El presente estudio tuvo como objetivo determinar la calidad de las grasas y aceites más consumidos por la población de nuestro país. Se recolectaron y codificaron 15 marcas diferentes de mantequillas o margarinas $(A, B, D 1$ a $D 11), 7$ tipos de mantecas $(\boldsymbol{E} \mathbf{1}$ a $\boldsymbol{E} 7)$ y 14 marcas diferentes de aceites de girasol (EG1 a $\boldsymbol{E} \boldsymbol{G} 3)$, maíz (EM1 a $\boldsymbol{E M 3})$, oliva $(\boldsymbol{E O} 1$ a $\boldsymbol{E O} 4)$, soya $(\boldsymbol{E S} 1$ a $\boldsymbol{E S} 3)$ y de palma africana (EV) (Cuadro 1). Un 67\% de los productos son elaborados por empresas nacionales y un $33 \%$ corresponden a productos importados y comercializados en nuestro país. Se determinó, mediante cromatografía de gases, la composición de los ácidos grasos, empleando resonancia magnética nuclear se calculó el número de dobles enlaces, hidrógenos alílicos y doblemente arílicos, también se analizaron los índices de yodo y saponificación, y la masa molar promedio en la fracción lipídica de muestras quintuplicadas de todos los productos. Utilizando estos parámetros, se encontró que 2 tipos de mantequillas y un tipo de aceite están probablemente adulterados con triacilgliceroles de origen diferente, ya que su composición no coincidió con la descripción en la etiqueta del producto. Considerando solamente el número de dobles enlaces, hidrógenos alílicos y doblemente arílicos presentes en los aceites, grasas y productos derivados, se determinó que los aceites de girasol, maíz y oliva son los más susceptibles a las reacciones de oxidación lipídica. A través de la determinación de la relación ácidos grasos poliinsaturados / ácidos grasos saturados y del índice de aterogenicidad se evaluó el potencial aterogénico de los distintos productos, se obtuvo como resultado que los más perjudiciales son 2 tipos de mantequillas y 5 tipos de mantecas.

Descriptores: Caracterización de grasas y aceites, aterogénico.

Escuela de Tecnología de Alimentos, Universidad de Costa Rica.

2. Escuela de Química, Universidad de Costa Rica.

Abreviaturas: RMN-H: resonancia magnética nuclear Correspondencia: Carlos Herrera Ramírez Escuela de Química, Universidad de Costa Rica

ISSN 0001-6002/2005/47/1/36-42 Acta Médica Costarricense, $\mathbb{O} 2005$ Colegio de Médicos y Cirujanos
Recibido: 11 de mayo de 2004

Aceptado: 5 de octubre de 2004

En los últimos años, los costarricenses hemos adquirido un estilo de vida propio de las ciudades modernas, caracterizado por la tensión nerviosa, el sedentarismo, la comida abundante y mal balanceada y los vicios, lo cual ha repercutido en el desarrollo de enfermedades del aparato circulatorio. La enfermedad más importante es la arterioesclerosis, caracterizada por producir un daño vascular generalizado con formación de ateromas en las arterias, oclusión parcial de la luz, produciendo disminución de la circulación sanguínea. Entre los principales factores que generan el desarrollo y el progreso de estas enfermedades, se citan: la hipercolesterolemia, los factores dietéticos, las altas concentraciones de lipoproteínas de baja densidad o bajas concentraciones de lipoproteínas de alta densidad, la hipertensión arterial, el tabaquismo, la obesidad, el sedentarismo, la tensión nerviosa y la predisposición genética. 
A pesar de la problemática nacional en el campo de las enfermedades cardiovasculares, poco se conoce sobre la composición lipídica de nuestros alimentos, por lo cual debe hacerse un esfuerzo por estimar el riesgo de la población ante la arteriosclerosis, tomando en cuenta las características alimentarias del costarricense.

Está ampliamente descrita la relación existente entre los niveles de colesterol plasmático y la arteriosclerosis: el colesterol se transporta en la sangre por las lipoproteínas de baja y alta densidad. Las lipoproteínas de baja densidad transportan cerca del 70\% del colesterol total del suero, mientras que las lipoproteínas de alta densidad están asociadas a la excreción del colesterol del cuerpo. Por lo tanto, niveles altos de éstas en la sangre, reducen el riesgo de este tipo de enfermedades, mientras que niveles elevados de lipoproteínas de baja densidad aumentan la probabilidad de adquirir la enfermedad, debido a que son oxidadas en el plasma sanguíneo, absorbidas por macrófagos y se depositan en la paredes arteriales internas, formando las placas ateromatosas $^{1,2}$.

Asociados a estas lipoproteínas, se encuentran los ácidos grasos que se ingieren en la dieta, los cuales pueden dividirse en ácidos saturados, monoinsaturados y poliinsaturados. Independientemente de la clasificación a que pertenezcan, los ácidos grasos difieren en el número de átomos de carbono y en el grado y tipo de insaturación que poseen, (cis o trans) lo cual determina su interacción en el organismo y su relación con el desarrollo de la arteriosclerosis. Los ácidos grasos saturados de cadena corta (10 carbonos o menos), debido a su solubilidad en agua, tienen un efecto neutro sobre el desarrollo de enfermedades cardiovasculares. Los ácidos laúrico, mirístico y palmítico enriquecen los fosfolípidos de las membranas celulares, interfiriendo con la función normal de los receptores de lipoproteínas de baja densidad, reduciendo su absorción y aumentando su concentración en el plasma, por lo que son considerados aterogénicos. El ácido esteárico se metaboliza a ácido oleico más rápidamente que otros ácidos grasos saturados, por lo tanto no es aterogénico. El ácido oleico tiene un efecto favorable en la prevención de las enfermedades cardiovasculares, debido a que reduce los niveles de colesterol total y de lipoproteínas de baja densidad. Los ácidos grasos poliinsaturados $\omega-6$ y $\omega$-3 tienen un efecto benéfico en la prevención de enfermedades del aparato circulatorio, debido a que reducen significativamente los niveles de colesterol total y de lipoproteínas de baja densidad en el plasma ${ }^{3-6}$.

Ulbricht y Southgate fijaron un índice al que denominaron índice de aterogenicidad, el cual se define como la razón del contenido de los ácidos grasos capaces de aumentar los niveles de colesterol sérico (ácidos láurico, mirístico y palmítico) y los ácidos grasos de acción protectora (mono y poliinsaturados) ${ }^{7}$.

\section{I.A. $=\frac{\text { ácido láurico }+4 \text { (ácido mirístico) }+ \text { ácido palmítico }}{\text { poliinsaturados } \omega-3+\text { poliinsaturados } \omega-6+\text { oleico }}$ \\ + otros monoinsaturados}

En el índice de aterogenicidad tiene más influencia el ácido mirístico, debido a la evidencia experimental, que lo señala como el principal promotor de los aumentos de colesterol sérico. En el denominador se incluyen todos los ácidos grasos que poseen una acción protectora, es decir aquellos que tienden a reducir el riesgo de desarrollo de enfermedades coronarias ${ }^{3-5,7}$. Los ácidos grasos monoinsaturados o poliinsaturados, que poseen la configuración trans tienen un comportamiento similar a los ácidos grasos saturados, por lo tanto son capaces de promover la formación de placas ateromatosas en las paredes arteriales ${ }^{6,8}$. Los argumentos anteriores evidencian la necesidad de controlar la ingesta de ácidos grasos y es debido a ello que la dieta se considera la piedra angular en la prevención de la arteriosclerosis, siendo de primordial importancia el realizar y adecuar estudios sobre la alimentación e incidencia de desórdenes cardiovasculares. Los esfuerzos para combatir estas enfermedades deben enfocarse en su prevención me-diante la identificación y control de los factores de riesgo ${ }^{9,10}$. Por tal razón, determinamos la composición de los ácidos grasos y así su relación con el potencial aterogénico de los aceites, grasas y otros derivados producidos o comercializados en Costa Rica.

El proceso de autooxidación o rancidez oxidativa de los aceites y grasas se lleva a cabo través de una serie de reacciones radicalarias con formación de hidroperóxidos como productos intermedios, éstos se descomponen para producir una gran variedad de compuestos volátiles, responsables del olor a rancio. La reacción de oxidación está influenciada por la composición de los ácidos grasos. La velocidad de este indeseable proceso es directamente proporcional a la cantidad de dobles enlaces, hidrógenos alílicos y doblemente alílicos presentes en los triacilgliceroles ${ }^{11,12}$, depende de la concentración y actividad de agentes pro y antioxidantes, de la presión parcial del oxígeno, de la superficie de contacto con el oxígeno y de las condiciones de almacenamiento (temperatura, luz, contenido acuoso) del aceite o grasa.

\section{Materiales y métodos}

\section{Recolección y tratamiento de las muestras.}

Con la colaboración de la Oficina Nacional de Normas y Unidades de Medida del Ministerio de Economía, Industria y Comercio y utilizando el modelo de muestreo preliminar ${ }^{13}$, se recolectaron muestras quintuplicadas de diferentes tipos y marcas de productos lipídicos, las cuales 
se identificaron de la siguiente manera mantequillas: $\boldsymbol{A}, \boldsymbol{B}$; margarinas: D1, D2, D3a, D3b, D4, D5, D6, D7, D8, D9a, $D 9 b, D 10, D 11$; grasas o mantecas: E1, E2, E3, E4, E5, E6, $\boldsymbol{E} 7$; aceites de girasol: $\boldsymbol{E} \boldsymbol{G 1}, \boldsymbol{E} \boldsymbol{G} 2, \boldsymbol{E} \boldsymbol{G 3}$; aceites de maíz: EM1, EM2, EM3; aceites de oliva: EO1, EO2, EO3, EO4; aceites de soya: $\boldsymbol{E S} 1, \boldsymbol{E S} 2, \boldsymbol{E S} 3$ y aceite de palma africana: $\boldsymbol{E} \boldsymbol{V}$, todos producidos o comercializados en nuestro país. Las muestras de un mismo tipo o marca se mezclaron para obtener una muestra compuesta, se codificaron en la forma indicada, se rotularon y se almacenaron en congelación para su análisis posterior.

\section{Extracción y cuantificación de aceites y grasas en las muestras.}

Utilizando metodología validada; se determinó por triplicado el contenido en porcentaje por masa / masa de agua y de triacilgliceroles presentes en cada una de las muestras emulsificadas (mantequillas y margarinas) ${ }^{12}$, (cuadro 1). La metodología utilizada para la determinación del contenido de agua en las muestras emulsificadas fue validada, obteniéndose una desviación estándar promedio de $0,50 \%$, como criterio de precisión y un $96 \%$ de recuperación, como criterio de exactitud.

\section{Identificación de ácidos grasos por cromatografía de gases.}

Se utilizó un cromatógrafo Perkin Elmer (modelo 9000), provisto de un detector de ionización de llama, de una columna capilar Supelcowax de $30 \mathrm{~m}$ de largo y $0,25 \mathrm{~mm}$ de diámetro. La temperatura del inyector y del detector fue de $220^{\circ} \mathrm{C}$ y $260^{\circ} \mathrm{C}$ respectivamente. En la columna, la temperatura inicial fue de $180^{\circ} \mathrm{C}$ por 0 minutos, con un gradiente de temperatura de $2^{\circ} \mathrm{C} / \mathrm{min}$ y una temperatura final de $240^{\circ} \mathrm{C}$ por 0 minutos. Las muestras de aceites y grasas se transesterificaron con hidróxido de tetrametilamonio al $25 \%$ en metanol anhidro, se inyectaron $(2-4 \mu \mathrm{L})$ en el cromatógrafo bajo las condiciones indicadas, se identificaron y se cuantificaron los ácidos grasos, utilizando como parámetro el tiempo de retención de los ácidos grasos presentes en los patrones, según el método de Metcalfe y Wang ${ }^{14}$.

Con la composición porcentual de los ácidos grasos determinada por esta técnica, se calculó el contenido total de ácidos grasos saturados, monoinsaturados y poliinsaturados. Además se hizo uso de los ácidos grasos indicadores para los diferentes productos, por ejemplo el ácido lino-leico, en una proporción entre 50 y 70\%, es el ácido graso indicador de los aceites de girasol, maíz, semilla de algodón y germen de trigo ${ }^{11}$.

Determinación del potencial de oxidación, índice de yodo, índice de saponificación y masa molar promedio de los aceites y grasas.

Con la composición de los ácidos grasos y haciendo uso de la técnica de espectroscopia de resonancia magnética

\begin{tabular}{|ccc|}
\hline \multicolumn{3}{|c|}{$\begin{array}{c}\text { Cuadro 1. Contenido (\% por } \mathrm{m} / \mathrm{m} \text { ) de } \\
\text { humedad y de triacilgliceroles en las muestras } \\
\text { emulsificadas }\end{array}$} \\
\hline (margarinas y mantequillas) \\
\hline Codigo & Agua & Triacilgliceroles \\
\hline A & 15,0 & 85 \\
B & 18,3 & 82 \\
D1 & 15,0 & 85 \\
D2 & 15,2 & 85 \\
D3a & 33,4 & 67 \\
D3b & 23,6 & 76 \\
D4 & 37,8 & 62 \\
D5 & 14,9 & 85 \\
D6 & 52,0 & 48 \\
D7 & 14,1 & 86 \\
D8 & 18,3 & 82 \\
D9a & 33,7 & 66 \\
D9b & 25,6 & 74 \\
D10 & 14,8 & 85 \\
D11 & ND & ND \\
\hline
\end{tabular}

nuclear (RMN- $\left.{ }^{1} \mathrm{H}\right)$, se calcularon los índices de yodo (centígramos de $\mathrm{I}^{2}$ por gramo de triacilglicerol), saponificación (miligramos de $\mathrm{KOH}$ por gramo de triacilglicerol) y la masa molar (gramos por mol) promedio de los aceites y grasas. Para evaluar la susceptibilidad de los aceites, grasas $\mathrm{u}$ otros derivados a las reacciones de oxidación, se determinó la cantidad de dobles enlaces, hidrógenos alílicos y doblemente alílicos presentes en las muestras recolectadas. También se determinó el contenido total de ácidos grasos $\omega$ 6 y $\omega-3^{16}$ (cuadro 3 ).

\section{Determinación de la relación poliinsaturados I saturados y el índice de aterogenicidad}

Se determinó la composición en porcentaje por masa / masa de ácidos grasos saturados, monoinsaturados y poliinsaturados, la relación poliinsaturados / saturados y el índice de aterogenicidad en cada una de las muestras recibidas (cuadro 4).

\section{Resultados}

En el cuadro 1, se muestran los resultados obtenidos para el contenido de humedad y de triacilgliceroles en las muestras emulsificadas (mantequillas y margarinas).

Las mantequillas ( $\boldsymbol{A}$ y $\boldsymbol{B})$ y la mayoría de las margarinas $(D 1, D 2, D 5, D 7, D 8$ y $D 10)$ cumplieron con estos requisitos; el grupo que presentó un contenido de grasa entre 62 y $76 \%$ (D3A, D3B, D4, D9A y $\boldsymbol{D} 9 \boldsymbol{B})$, puede considerarse como formado por margarinas con un valor energético reducido, pero no como margarinas livianas o dietéticas. 


\begin{tabular}{|c|c|c|c|c|c|c|}
\hline \multicolumn{7}{|c|}{$\begin{array}{l}\text { Cuadro 2: Ámbito de variación del índice de yodo (I.I.), índice de saponificación (I.S.) } \\
\text { masa molar (M.M.) y cantidad de dobles enlaces (D.E.), hidrógenos alílicos (H.A.) y } \\
\text { doblemente alílicos (H.D.A.) en los aceites, grasas y productos derivados. }\end{array}$} \\
\hline CODIGO & $\begin{array}{c}\text { I.I. } \\
\text { (cg Yodo/g) }\end{array}$ & $\begin{array}{c}\text { I.S. } \\
\text { (mg KOH/g) }\end{array}$ & $\begin{array}{c}\text { M.M. } \\
\text { (g/mol) }\end{array}$ & D.E. & H.A. & H.D.A. \\
\hline$A$ & $32-37$ & $206-209$ & $804-815$ & 1,0 & 4,0 & 0,2 \\
\hline B & $33-41$ & $205-210$ & $799-818$ & 1,3 & 4,5 & 0,4 \\
\hline $\mathrm{D} 1$ & $74-83$ & $169-190$ & 884-992 & 2,9 & 8,3 & 1,6 \\
\hline D2 & $56-63$ & $174-191$ & $878-963$ & 2,2 & 7,4 & 0,7 \\
\hline D3a & $97-102$ & $162-190$ & $884-1034$ & 3,6 & 9,1 & 2,5 \\
\hline D3b & $53-61$ & $176-192$ & $876-954$ & 2,1 & 7,1 & 0,7 \\
\hline D4 & $54-66$ & $173-191$ & $878-974$ & 2,3 & 7,6 & 0,7 \\
\hline D5 & $59-67$ & $172-191$ & $879-977$ & 2,3 & 7,6 & 0,8 \\
\hline D6 & $102-107$ & $164-189$ & $890-1022$ & 3,8 & 9,5 & 2,8 \\
\hline D7 & $98-108$ & $167-189$ & 890-1007 & 3,8 & 9,5 & 2,8 \\
\hline D8 & $106-115$ & $167-189$ & $890-1009$ & 4,0 & 10,0 & 3,1 \\
\hline $\mathrm{D} 9 \mathrm{a}$ & $105-114$ & $169-189$ & 891-994 & 4,0 & 9,8 & 3,1 \\
\hline $\mathrm{D} 9 \mathrm{~b}$ & $87-105$ & $165-188$ & 893-1017 & 3,7 & 9,9 & 2,4 \\
\hline D10 & $52-60$ & $174-192$ & $877-967$ & 2,1 & 7,1 & 0,6 \\
\hline D11 & $99-112$ & $110-188$ & $894-1524$ & 4,0 & 10,5 & 2,7 \\
\hline E1 & $55-62$ & 174-198 & $847-966$ & 2,1 & 6,2 & 1,0 \\
\hline E2 & $45-52$ & $175-194$ & $866-960$ & 1,8 & 5,8 & 0,6 \\
\hline E3 & $47-50$ & 173-195 & $863-970$ & 1,7 & 5,7 & 0,6 \\
\hline E4 & $85-91$ & $164-189$ & $890-1023$ & 3,2 & 8,7 & 2,0 \\
\hline E5 & $43-54$ & $172-194$ & $866-979$ & 1,8 & 6,1 & 0,6 \\
\hline E6 & $43-51$ & $174-193$ & $870-967$ & 1,7 & 5,9 & 0,6 \\
\hline E7 & $9-15$ & $228-239$ & $703-737$ & 0,4 & 1,6 & 0,1 \\
\hline EG1 & $129-133$ & $182-188$ & $894-924$ & 4,7 & 10,9 & 3,9 \\
\hline EG2 & $130-134$ & $185-188$ & 894-908 & 4,7 & 10,8 & 4,0 \\
\hline EG3 & $126-134$ & $174-188$ & $893-967$ & 4,7 & 10,5 & 4,1 \\
\hline EM1 & $124-126$ & 188-189 & $890-894$ & 4,4 & 10,5 & 3,6 \\
\hline EM2 & $119-127$ & $178-189$ & $890-948$ & 4,5 & 10,3 & 3,8 \\
\hline EM3 & $127-134$ & $188-196$ & 857-892 & 4,4 & 10,5 & 3,6 \\
\hline EO1 & $78-79$ & $184-188$ & $893-914$ & 2,7 & 10,2 & 0,4 \\
\hline EO2 & $70-78$ & $185-188$ & 894-909 & 2,7 & 10,3 & 0,3 \\
\hline EO3 & $73-79$ & $162-188$ & 894-1039 & 2,8 & 10,3 & 0,4 \\
\hline EO4 & $76-79$ & $187-188$ & $891-897$ & 2,8 & 10,1 & 0,5 \\
\hline ES1 & $120-126$ & $185-188$ & $891-908$ & 4,4 & 10,2 & 3,8 \\
\hline ES2 & $121-124$ & $188-189$ & $890-892$ & 4,4 & 10,1 & 3,7 \\
\hline ES3 & $125-128$ & $188-189$ & $890-891$ & 4,5 & 10,2 & 3,9 \\
\hline EV & $95-97$ & $189-191$ & $880-891$ & 3,4 & 8,4 & 2,5 \\
\hline
\end{tabular}

Utilizando la técnica de cromatografía de gases se determinó la composición de los ácidos grasos en los diferentes aceites, grasas y en la fracción lipídica de los productos emulsificados (mantequillas y margarinas).

Usualmente, para interpretar la composición de los ácidos grasos, se hace uso de los ácidos grasos indicadores, los cuales son característicos de la clase de aceite o grasa, pudiendo inclusive ser utilizados como indicadores de adulteración ${ }^{11}$. La grasa láctea debe presentar una composición porcentual de ácido butírico cercana a 3,7\%, ya que éste es su ácido graso indicador; sin embargo, los productos $\boldsymbol{A}$ y $\boldsymbol{B}$ elaborados con este tipo de grasa, presentaron un contenido de ácido butírico entre 1,7 y 1,8\%. Las mantequillas $\boldsymbol{A}$ y $\boldsymbol{B}$ presentaron un alto contenido de ácidos grasos aterogéni- cos, entre 3,8-2,5\% de ácido láurico; 9,1-9,5\% de ácido mirístico y $26,5-28,5 \%$ de ácido palmítico.

La grasa usada en la elaboración de las margarinas (D1 a D11) no pudo ser identificada utilizando el criterio de los ácidos grasos indicadores, debido a que era una mezcla de aceites o grasas o fue hidrogenada en forma parcial.

Las mantecas $\boldsymbol{E} 1, \boldsymbol{E} 2, \boldsymbol{E} 3, \boldsymbol{E} 5$ y $\boldsymbol{E} 6$ presentaron la composición típica de la grasa extraída del fruto de la palma africana (Elaeis guineensis), la cual se caracteriza por un contenido de ácido palmítico entre 40 y 45\%, de ácido oleico cercano a $40 \%$, de ácido linoleico alrededor de $10 \%$ y de ácido mirístico un 1\%. El producto $\boldsymbol{E} 4$ es una manteca importada, constituida por una mezcla de aceites o grasas hidrogenados parcialmente. 


\begin{tabular}{|c|c|c|c|c|c|}
\hline \multicolumn{6}{|c|}{$\begin{array}{l}\text { Cuadro 3: Contenido ( } \% \text { por } \mathrm{m} / \mathrm{m} \text { ) promedio } \\
\text { de ácidos grasos saturados, monoinsaturados, } \\
\text { poliinsaturados, } \omega-6 \text { y } \omega-3 \text { en los productos } \\
\text { analizados. }\end{array}$} \\
\hline Código & Saturados & $\begin{array}{l}\text { Monoin- } \\
\text { saturados }\end{array}$ & $\begin{array}{l}\text { Poliin- } \\
\text { saturados }\end{array}$ & $\begin{array}{c}\text { Acidos } \\
\text { grasos } \\
\omega-6\end{array}$ & $\begin{array}{c}\text { Acidos } \\
\text { grasos } \\
\omega-3\end{array}$ \\
\hline$A$ & 62,6 & 33,0 & 3,1 & 0,5 & 2,6 \\
\hline$B$ & 61,5 & 31,9 & 5,6 & 0,8 & 4,8 \\
\hline D1 & 30,6 & 44,6 & 24,9 & 2,5 & 22,4 \\
\hline $\mathrm{D} 2$ & 38,3 & 51,3 & 10,4 & 0,6 & 9,9 \\
\hline D3a & 23,9 & 37,9 & 38,2 & 0,6 & 10,0 \\
\hline D3b & 41,1 & 48,2 & 10,7 & 4,2 & 34,0 \\
\hline D4 & 36,3 & 52,3 & 11,4 & 0,7 & 10,7 \\
\hline D5 & 36,6 & 50,9 & 12,6 & 0,9 & 11,7 \\
\hline D6 & 20,7 & 37,9 & 41,4 & 4,9 & 36,5 \\
\hline D7 & 20,6 & 37,6 & 41,8 & 4,8 & 36,9 \\
\hline D8 & 17,1 & 36,5 & 46,4 & 5,6 & 40,8 \\
\hline D9a & 18,7 & 34,8 & 46,5 & 5,5 & 41,0 \\
\hline D9b & 17,3 & 46,6 & 36,2 & 4,4 & 31,7 \\
\hline D10 & 41,2 & 49,1 & 9,6 & 0,7 & 9,0 \\
\hline D11 & 12,5 & 50,7 & 36,8 & 7,6 & 29,2 \\
\hline $\mathrm{E} 1$ & 45,9 & 35,0 & 16,5 & 0,3 & 13,0 \\
\hline E2 & 51,6 & 38,2 & 10,5 & nd & 10,1 \\
\hline E3 & 52,8 & 38,0 & 9,2 & nd & 9,2 \\
\hline E4 & 27,5 & 41,0 & 31,5 & 2,2 & 29,3 \\
\hline E5 & 51,3 & 38,2 & 10,4 & nd & 10,4 \\
\hline E6 & 52,0 & 39,6 & 9,2 & nd & 9,2 \\
\hline E7 & 86,9 & 12,2 & 0,9 & 0,3 & 0,7 \\
\hline EG1 & 9,5 & 24,8 & 65,7 & 0,1 & 65,5 \\
\hline EG2 & 10,0 & 24,7 & 66,8 & nd & 66,8 \\
\hline EG3 & 12,3 & 22,0 & 66,8 & 1,9 & 64,9 \\
\hline EM1 & 12,4 & 30,5 & 58,6 & 0,9 & 57,6 \\
\hline EM2 & 14,2 & 28,3 & 59,2 & 3,8 & 55,3 \\
\hline EM3 & 15,8 & 27,6 & 58,5 & 1,4 & 57,6 \\
\hline E01 & 14,6 & 80,4 & 5,5 & 0,5 & 5,0 \\
\hline EO2 & 14,4 & 76,4 & 5,1 & 0,6 & 4,5 \\
\hline EO3 & 13,9 & 81,1 & 5,7 & 0,5 & 5,2 \\
\hline EO4 & 15,6 & 79,9 & 8,0 & 0,6 & 7,3 \\
\hline ES1 & 15,3 & 27,6 & 56,3 & 6,5 & 49,8 \\
\hline ES2 & 15,8 & 29,5 & 55,0 & 6,5 & 49,8 \\
\hline ES3 & 15,4 & 28,3 & 58,2 & 6,5 & 49,8 \\
\hline EV & 29,6 & 28,7 & 37,6 & 3,8 & 33,8 \\
\hline
\end{tabular}

De las grasas analizadas, el aceite de coquito contenía la mayor proporción de ácidos grasos aterogénicos: 44,2\% de ácido láurico, $14,3 \%$ de ácido mirístico y 8,9\% de ácido palmítico.

Los aceites de girasol $\boldsymbol{E} \boldsymbol{G} \mathbf{1}, \boldsymbol{E} \boldsymbol{G} \mathbf{2}$ y $\boldsymbol{E} \boldsymbol{G} \mathbf{3}$ y los aceites de maíz $\boldsymbol{E M 1 , ~} \boldsymbol{E M} 2$ y $\boldsymbol{E M} 3$ cumplieron con el contenido de ácidos grasos indicadores. Estos aceites poseían entre 5,9 y 10,6\% de ácido palmítico, un ácido graso aterogénico; pero también contenían una alta proporción de ácido oleico y de ácido linoleico, ambos con reconocida acción protectora sobre el desarrollo de enfermedades cardiovasculares.

Los aceites de oliva EO1, EO2, EO3 y EO4 presentaron un elevado contenido de ácido oleico (75-80\%), el

\begin{tabular}{|c|c|c|}
\hline \multicolumn{3}{|c|}{$\begin{array}{l}\text { Cuadro 4. Índice de aterogenicidad (I.A.) } \\
\text { y relación poliinsaturados / saturados (P/S) } \\
\text { promedio de aceites, grasas y derivados. }\end{array}$} \\
\hline CODIGO & I.A. & P/S \\
\hline A & 1,7 & 0,0 \\
\hline B & 1,5 & 0,1 \\
\hline D1 & 0,3 & 0,8 \\
\hline $\mathrm{D} 2$ & 0,4 & 0,2 \\
\hline D3a & 0,2 & 1,1 \\
\hline D3b & 0,4 & 0,2 \\
\hline D4 & 0,3 & 0,2 \\
\hline D5 & 0,4 & 0,3 \\
\hline D6 & 0,1 & 1,0 \\
\hline D7 & 0,1 & 1,7 \\
\hline D8 & 0,1 & 2,2 \\
\hline D9a & 0,1 & 1,6 \\
\hline D9b & 0,1 & 1,6 \\
\hline D10 & 0,5 & 0,2 \\
\hline D11 & 0,1 & 2,9 \\
\hline E1 & 0,9 & 0,4 \\
\hline E2 & 1,0 & 0,2 \\
\hline E3 & 1,1 & 0,2 \\
\hline E4 & 0,2 & 1,1 \\
\hline E5 & 1,0 & 0,2 \\
\hline E6 & 1,0 & 0,2 \\
\hline E7 & 8,4 & 0,0 \\
\hline EG1 & 0,1 & 6,9 \\
\hline EG2 & 0,1 & 6,8 \\
\hline EG3 & 0,1 & 5,4 \\
\hline EM1 & 0,1 & 4,7 \\
\hline EM2 & 0,1 & 4,2 \\
\hline EM3 & 0,1 & 4,8 \\
\hline EO1 & 0,1 & 0,4 \\
\hline EO2 & 0,1 & 0,4 \\
\hline EO3 & 0,1 & 0,4 \\
\hline EO4 & 0,1 & 0,5 \\
\hline ES1 & 0,1 & 3,7 \\
\hline ES2 & 0,1 & 3,5 \\
\hline ES3 & 0,1 & 3,8 \\
\hline EV & 0,4 & 1,3 \\
\hline
\end{tabular}

ácido indicador para este tipo de aceite y con efecto benéfico en la prevención de enfermedades del aparato circulatorio ${ }^{11}$.

El ácido graso indicador de los aceites de soya, es el ácido linolénico (9-10\%); sin embargo los aceites de soya $\boldsymbol{E S} 1, \boldsymbol{E S} 2$ y $\boldsymbol{E S} 3$ presentaron un 6,5\% de este ácido graso. El aceite $\boldsymbol{E} \boldsymbol{V}$, según la etiqueta, es elaborado con aceite de palma; pero no presentó la composición típica del aceite extraído del fruto de Elaeis guineensis " Contenía pequeñas cantidades de ácido láurico $(0,2 \%)$ y de ácido mirístico $(0,6 \%)$ y una proporción elevada de ácido palmíti$\operatorname{co}(24,3 \%)$. 
Según se muestra en el cuadro 2 el índice de saponificación de $\boldsymbol{A}$ y $\boldsymbol{B}$ fue significativamente menor (95\% de confianza) que el valor reportado en la literatura para la grasa láctea (210-230 mg KOH/g) ${ }^{17}$, debido probablemente a una adulteración con aceites o grasas de mayor masa molar.

El índice de yodo y el índice de saponificación de las mantecas $\boldsymbol{E} 1, \boldsymbol{E} 2, \boldsymbol{E} 3, \boldsymbol{E} 5$ y $\boldsymbol{E} \boldsymbol{6}$ coincidió con los valores reportados en la literatura para el aceite de palma africana. La manteca $\boldsymbol{E} 7$ presentó un índice de saponificación dentro del ámbito reportado en la literatura (220-235 mg $\mathrm{KOH} / \mathrm{g}$ ) para el aceite de coquito ${ }^{11,17}$.

Los índices de saponificación y de yodo de los aceites de girasol, maíz, oliva y soya no fueron significativamente diferentes ( $95 \%$ de confianza) de los valores reportados en la literatura ${ }^{11,17}$.

Los aceites de girasol, maíz y soya fueron los productos más susceptibles a las reacciones de oxidación, presentando entre 4 y 5 dobles enlaces, entre 10 y 11 hidrógenos alílicos y 4 hidrógenos doblemente alílicos. Los aceites de oliva fueron más estables a la oxidación, ya que presentaron 3 dobles enlaces y 10 hidrógenos alílicos solamente. Las margarinas $D 3 a, D 6, D 7, D 8, D 9 a, D 9 b$ y $D 11$ presentaron entre 3 y 4 dobles ligaduras, entre 9 y 10 hidrógenos alílicos y entre 2 y 3 hidrógenos doblemente alílicos. La menor susceptibilidad a las reacciones de oxidación, la presentaron $\boldsymbol{A}, \boldsymbol{B}$ y $\boldsymbol{E} 7$ y con susceptibilidad intermedia, los productos restantes.

En el cuadro 4 se presentan los valores promedios encontrados para el índice de aterogenicidad y la relación poliinsaturados / saturados de cada una de las muestras recolectadas.

\section{Discusión}

Se determinó la composición de los ácidos grasos, los índices de yodo, saponificación, la masa molar promedio, el número de dobles enlaces, hidrógenos alílicos y doblemente alílicos en la fracción lipídica de muestras quintuplicadas de 15 marcas diferentes de mantequillas o margarinas, 7 tipos de mantecas y 14 marcas diferentes de aceites de girasol, maíz, oliva, soya y de palma africana. También se evaluó el potencial aterogénico de los productos a través de la determinación de la relación ácidos grasos poliinsaturados / ácidos grasos saturados e índice de aterogenicidad .

Las margarinas y mantequillas deben contener como mínimo un $80 \%$ de triacilgliceroles, $18 \%$ de agua y un $2 \%$ de otros componentes: emulsificantes (0,50-1,0\%), ácido cítrico o láctico (para ajustar el $\mathrm{pH}$ a 4,2-4,5), sal común (0,1-0,2\%), sustancias aromáticas y pigmentos ${ }^{11}$. La literatura indica que las margarinas livianas deben contener una cantidad de grasa menor a $40 \%$, mayor cantidad de agua y de agentes tensoactivos, por lo que solamente la margarina D6 cumplió con estas características ${ }^{11}$.

Se encontró que algunos de los productos fueron probablemente adulterados y otros eran mezclas de diferentes tipos de aceites o grasas y no había correspondencia con su etiqueta: las mantequillas $\boldsymbol{A}$ y $\boldsymbol{B}$ indican una adulteración con aceites o grasas de diferente origen, las grasas de las margarinas D1 a D11 corresponden a mezclas de aceites y grasas probablemente hidrogenados en forma parcial. Debido a ello los índices de yodo y saponificación no se compararon con los de la literatura, porque se desconoce la identidad del aceite o grasa presente en estos productos.

El aceite $\boldsymbol{E} \boldsymbol{V}$ no presentó la composición típica de aceite de palma, que se establece en la etiqueta.

En las mantecas $\boldsymbol{E} 1, \boldsymbol{E} 2, \boldsymbol{E} 3, \boldsymbol{E} 5$ y $\boldsymbol{E} 6$ la proporción de ácido palmítico fue muy elevada; por lo que no se recomienda su consumo; ya que este ácido graso promueve la formación de ateromas en los arteriales.

De las grasas analizadas, la manteca $\boldsymbol{E} 7$ contenía la mayor proporción de ácidos grasos aterogénicos, por lo que se desaconseja el consumo directo o indirecto de este producto; ya que estos ácidos grasos contribuyen al desarrollo de enfermedades del aparato circulatorio. La manteca $\boldsymbol{E} 7$ es una grasa rica en ácido laúrico (45\%), que es el ácido graso indicador de la grasa de las semillas del fruto de la palma africana (Elaeis guineensis) y es conocida en Costa Rica, como aceite de coquito.

El aceite $\boldsymbol{E} \boldsymbol{V}$, posee una proporción elevada de ácido palmítico por lo que no se recomienda su uso en la preparación de los alimentos.

Las mantequillas $\boldsymbol{A}$ y $\boldsymbol{B}$ presentaron un alto contenido de ácidos grasos aterogénicos, por lo cual no es recomendable su consumo.

Los productos más aterogénicos son las mantequillas, lactocremas y algunas de las marcas de manteca. Los aceites con menor índice de aterogenicidad presentan menor posibilidad de formación de ateromas, destacándose los aceites de girasol, maíz y oliva. Con respecto a la relación poliinsaturados / saturados son los aceites de girasol, maíz y soya los que presentan la relación más elevada. Para prevenir el desarrollo de enfermedades cardiovasculares se recomienda el consumo moderado de estos aceites; en orden de preferencia los de girasol, maíz, oliva y soya.

Los productos más susceptibles a las reacciones de oxidación fueron los aceites de girasol, maíz y oliva, los cuales deben de protegerse con antioxidantes adecuados, envasarse al vacío (exclusión de oxígeno) y en recipientes que no permitan el paso de la luz solar o artificial ${ }^{11}$. 


\section{Agradecimiento}

A la Vicerrectoría de Investigación de la Universidad de Costa Rica por el financiamiento parcial del proyecto $\mathrm{N}^{\mathrm{o}}$ 115-AO-159: “Potencial aterogénico de aceites, grasas y productos derivados producidos o comercializados en Costa Rica".

A la Oficina Nacional de Normas y Unidades de Medida, del Ministerio de Economía, Industria y Comercio por realizar el muestreo de aceites, grasas y otros productos derivados.

A la Escuela de Química, de la Universidad de Costa Rica por el apoyo dado a este proyecto en infraestructura, equipo y materiales de laboratorio.

Abstract

Cardiovascular deseases are very common among Costa Rican people. They are related to diets high in lipids that cause arterial damage. The present study was undertaken to determine the quality of fats and oils consumed more frequently in our country. 15 different brands of butter and margarines $(A, B, D 1$ a $D 11), 7$ types of vegetable fat $(\boldsymbol{E} 1$ a $\boldsymbol{E} 7)$ and 14 different brands of sunflower oil (EG1 a $E G 3)$, corn oil (EM1 a $E M 3)$, olive oil (EO1 a EO4), soy oil (ES1 a ES3) and palm oil $(\boldsymbol{E V})$ (Fig. 1) were collected and identified 67 percent of the products were made in Costa Rica, 33\% were imported products. Using gas chromatography and nuclear magnetic resonance techniques, fatty acid composition, iodine and saponification number, average molecular weight, carboncarbon double bond number, allylic and double allylic hydrogens were determined in the lipid fraction of the 36 different products. Two types of butter and one type of oil were found adulterated with triacylglycerols of different kind or source. Susceptibility of the products to lipid oxidation was determined only in terms of double bond number and allylic and double allylic hydrogens. Sunflower, corn and olive oils were the most susceptible products. Through polyunsaturated fatty acids / saturated fatty acids relation and atherogenic index the atherogenic potencial of the products was evaluated. The findings were that 2 types of butter and 5 types of vegetable fat were the most injurious ones.

\section{Referencias}

1. Regnstrom J, Nilsson J, Tornvall P, Landou C, Hamsten, A Susceptibility to low-density lipoprotein oxidation and coronary atherosclerosis in man. Lancet 1992; 339: 1183-1186.

2. Libby, P. Una nueva teoría sobre la aterosclerosis. Investigación y ciencia. 2002. Julio: 15-23.

3. Woollett AL, Spady K.D, Dietschy MJ, Saturated and unsaturated fatty acids independently regulate low-density lipoprotein receptor activity and production rate. J Lipid Res. 1992; 33: 77-88.

4. Hayes KC, Pronczuk A, Lindsay S, Diersen-Scahde D. Dietary saturated fatty acids $(12: 0,14: 0,16: 0)$ differ in their impact on plasma cholesterol and lipoproteins in nonhuman primates. Am J Clin Nutr 1991. 53: 491-498.

5. Grundy M.S. Which Saturated Fatty Acids Raise Plasma Cholesterol Levels?. 1989: 83-93.

6. Grundy, M.S. Trans monounsaturated fatty acids and serum cholesterol levels. N Engl J Med. 1990; 323: 480-481.

7. Ulbricht TLV, Southgate DAT. Coronary heart disease: seven dietary factors. Lancet. 1991; 338: 985-992.

8. Katan MB. Los ácidos grasos trans y las lipoproteínas del plasma. Nutrition Reviews. 2001; 2: 21-25.

9. Acuña M. Estudio del potencial aterogénico y el contenido de colesterol en productos grasos de consumo popular en Costa Rica. Tesis de Licenciatura en Química. UCR. San José. 1995.

10. Jiménez M. Factores de riesgo coronario: estrategias para la prevención de la enfermedad coronaria. Rev Cost Cienc Med. 1990; 11: 31 40 .

11. Belitz H.D, Grosch W. Química de los alimentos. (2 ed). Acribia, S.A Zaragoza. 1992: 211-241, 691-723.

12. Matissek R, Schnepel F-M, Steiner G. Análisis de los alimentos: fundamentos, métodos y aplicaciones. Acribia. Zaragoza. 1992: 8, 9, 44-81.

13. Ministerio de Economía, Industria y Comercio. Ley $\mathrm{N}^{\circ} 7472$. Ley de la promoción de la competencia y defensa efectiva del consumidor y su reglamento. Decreto $\mathrm{N}^{\circ}$ 25234-MEIC. 1995.

14. Metcalfe L.D, Wang CN, Rapid preparation of fatty acid methyl esters using organic base-catalyzed transesterification. J Chromatog Sci . 981; 19: 530-535.

15. Nathan J. Resonancia magnética nuclear de hidrógeno. Programa regional de desarrollo científico y tecnológico. OEA. Washington, D.C. 1973: 78-80.

16. Sacchi R, Proton nuclear magnetic resonance rapid and structure specific determination of $\mathrm{w}-3$ polyunsaturated fatty acids in fish lipids. J Am Oil Chem Soc. 1993. 70; 225-228.

17. Dean JA. Lange's Handbook of Chemistry. (13 ed) Mc Graw Hill. New York. 1985. 7:724-731. 